CORRECTION

https://doi.org/10.1038/s41586-019-1123-5

\title{
Author Correction: Attosecond angular streaking and tunnelling time in atomic hydrogen
}

U. Satya Sainadh, Han Xu, Xiaoshan Wang, A. Atia-Tul-Noor, William C. Wallace, Nicolas Douguet, Alexander Bray, Igor Ivanov, Klaus Bartschat, Anatoli Kheifets, R. T. Sang \& I. V. Litvinyuk

Correction to: Nature https://doi.org/10.1038/s41586-019-1028-3, published online 18 March 2019.

In this Letter, the statement 'I.I. and A.B. performed their computations at the NCI Australia' was missing from the Acknowledgements section. The original Letter has been corrected online. 